\title{
Vibration Damping Control for Mobile Scaffolding Device Based on Skyhook Damper Theory
}

\author{
Katutoshi Kodama, Akihiro Morinaga, Yoichi Shimomoto, \\ Ikuo Yamamoto, and Hiroshi Matuda \\ Graduate School of Engineering, Nagasaki University, 1-14 Bunkyo, Nagasaki 852-8521, Japan
}

(Received November 30, 2020; accepted February 17, 2021)

Keywords: infrastructure inspection, wired control, semi-active dynamic absorber, skyhook theory

We are currently developing a mobile scaffolding device that can dodge the piers of bridges without dismantling the scaffold when moving between spans to improve efficiency and safety in bridge inspections. The mobile scaffolding device treated in this paper is split in the center of the device when it crosses the piers. In this process, the mobile scaffold is cantilevered and poses a danger to the workers on board. Therefore, we implemented horizontal holding control by wire reeling to keep the mobile scaffold horizontal. However, in this control method, the direction of motion of the mobile scaffold is only partially constrained by the wire. Thus, the device is greatly excited when an external force is applied. It is difficult to damp these oscillations in wire-reeling control. We designed a semi-active dynamic absorber for vibration control by applying the skyhook theory, a basic theory of the active suspension. When a random input that simulates the wind force was given to the mobile scaffolding device with the dynamic absorber, the vibration of the device was reduced by half compared with that of the mobile scaffolding device without the dynamic absorber.

\section{Introduction}

Typical bridge inspection methods in Japan are rope access, ${ }^{(1)}$ in which workers hang from ropes attached to the underside of a bridge, inspection using a bridge inspection vehicle, ${ }^{(2)}$ and rack scaffolding, ${ }^{(3)}$ in which two rack rails attached to the underside of a bridge are used as a track. In rope access, workers are suspended by lifelines only, which poses a risk of falling and other hazards, and work efficiency is dependent on worker proficiency. When using a bridge inspection vehicle, traffic control is necessary because the vehicle travels slowly while performing the inspection. When using rack scaffolding, the scaffolding needs to be dismantled and reassembled for each pier on a multispan bridge. In addition, even if the rack scaffold is capable of "pass-pier" over piers while turning, the scaffold has few support points, which makes it dangerous for workers to swing when turning.

Currently, we are developing a mobile scaffolding device similar to that in Fig. 1, which can circumvent the piers safely without dismantling the scaffold after the inspection of the span

*Corresponding author: e-mail: bb52119119@ms.nagasaki-u.ac.jp

https://doi.org/10.18494/SAM.2021.3206 


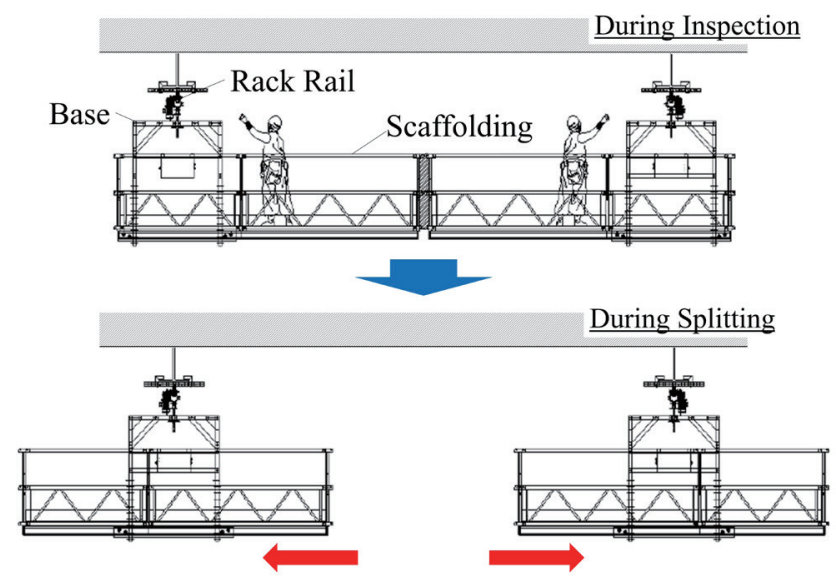

Fig. 1. (Color online) Mobile scaffolding device.

to solve the problems of bridge inspection mentioned above. The mobile scaffolding device we consider in this paper consists of two connected scaffolds that move along rails laid on the underside of the bridge. After reaching the piers, the device is divided by the operator by sliding the scaffolding part of the device against the abutment part. Each of the segmented devices performs pass-pier independently. The scaffold is cantilevered on the rail and poses a danger to the workers on board.

We previously proposed a horizontal holding control method using wire-reeling to hold this cantilevered mobile scaffold horizontally. ${ }^{(4,5)}$ However, since this wire restricts the scaffold to only a part of the direction of motion, the scaffold swings significantly in the direction of the unrestrained motion when it is subjected to external forces such as wind. In this paper, a semiactive dynamic absorber ${ }^{(6)}$ is implemented on a mobile scaffolding device to reduce the sway of the mobile scaffolding device due to external forces.

This paper is organized as follows. In Sect. 2, we summarize the mathematical model of the mobile scaffold, which is the control object of this paper. Section 3 discusses horizontal holding control for a mobile scaffolding device without a dynamic absorber. Section 4 proposes a design method for a semi-active dynamic absorber and evaluates the designed dynamic absorber using the proposed method. Section 5 concludes this study.

\section{Modeling of the Controlled Object}

Figure 2 shows the model of the mobile scaffolding device, which is the controlled object of this paper, and the coordinate system defined for the device. The control system treated in this paper consists of a horizontal holding mechanism (wire-reeling device) that holds the scaffold horizontally, a reaction force generator that cancels the scaffold directional component of the wire tension generated by the horizontal holding control, and a semi-active dynamic absorber that is used for damping control of the scaffold. 


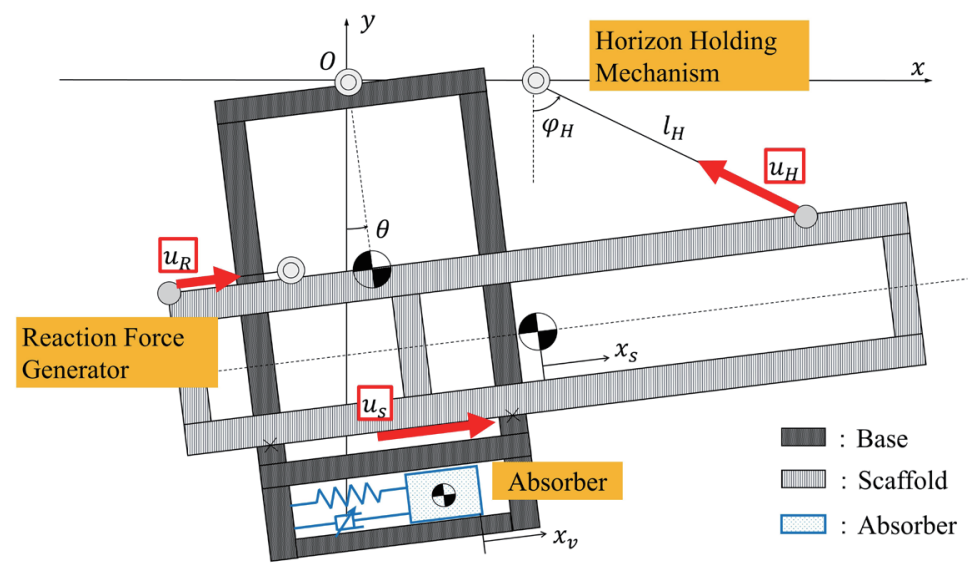

Fig. 2. (Color online) Model diagram of mobile scaffolding device.

\subsection{Parameters of mobile scaffolding device}

In Fig. 2, $\theta[\mathrm{rad}]$ is the tilt of the mobile scaffolding device, $x_{S}[\mathrm{~m}]$ is the amount of segmental movement of the scaffold, $x_{v}[\mathrm{~m}]$ is the displacement of the semi-active dynamic absorber, $l_{H}[\mathrm{~m}]$ is the wire length of the horizontal holding mechanism, and $\varphi_{H}[\mathrm{rad}]$ is the angle between the $y$-axis and the wire. $u_{s}$ is the input for the scaffold division operation, $u_{H}$ is the input for wire reeling in the horizontal holding mechanism, and $u_{R}\left(=-\sin \left(\varphi_{H}-\theta\right) u_{H}\right)$ is the force generated by the reaction force generator.

The system diagram of the scaffold division operation and the horizontal holding mechanism of the mobile scaffolding device is shown in detail in Fig. 3. The tilt of the mobile scaffolding device $(\theta)$ is measured by a tilt sensor. The amount of segmental movement of the scaffold $\left(x_{s}\right)$ is calculated from the number of pulses of the rotary encoder attached to the linear actuator. The wire length $\left(l_{H}\right)$ is calculated from the number of pulses of the rotary encoder attached to the dc motor. The physical parameters of the controlled object are shown in Table 1. The moments of inertia $I_{1}$ of the girders and $I_{2}$ of the scaffolding are given by

$$
I_{1}=\frac{m_{1}}{12}\left(a_{1}^{2}+b_{1}^{2}\right)+m_{2}\left(l_{1 x}^{2}+l_{1 y}^{2}\right), I_{2}=\frac{m_{2}}{12}\left(a_{2}^{2}+b_{2}^{2}\right)+m_{2}\left\{\left(l_{2 x}+x_{s}\right)^{2}+l_{2 y}^{2}\right\} .
$$

\subsection{Equation of motion for mechanical systems}

With the generalized coordinates $\boldsymbol{q}=\left[\theta, x_{S}, x_{v}, l_{H}, \varphi_{H}\right]^{T}$ and the input vector $\boldsymbol{u}=\left[u_{S}, u_{H}\right]^{T}$, the equation of motion for the mobile scaffolding device in Fig. 3 is given by

$$
M(q) \ddot{q}+V(q, \dot{q}) \dot{q}+G(q)+K(q)+D \dot{q}=B(q) u-A(q)^{T} \lambda
$$

where $\boldsymbol{M}(\boldsymbol{q}) \in \mathbb{R}^{5 \times 5}$ is the inertia matrix, $\boldsymbol{V}(\boldsymbol{q}, \dot{\boldsymbol{q}}) \in \mathbb{R}^{5 \times 5}$ is the matrix representing the Coriolis force and centrifugal force, $\boldsymbol{G}(\boldsymbol{q}) \in \mathbb{R}^{5}$ is the gravity vector, $\boldsymbol{K}(\boldsymbol{q}) \in \mathbb{R}^{5}$ is the elastic force 


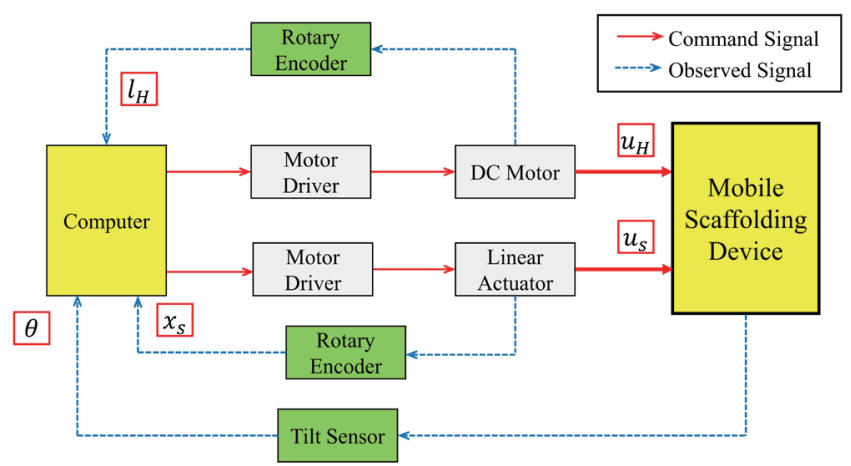

Fig. 3. (Color online) System diagram of mobile scaffolding device.

Table 1

Physical parameters of mobile scaffolding device (nominal values).

\begin{tabular}{lcc}
\hline & Parameter & Value \\
\hline$m_{1}$ & Mass of base & $3.4 \mathrm{~kg}$ \\
\hline$m_{2}$ & Mass of scaffold & $6.0 \mathrm{~kg}$ \\
\hline$a_{1}$ & Width of base & $0.56 \mathrm{~m}$ \\
\hline$b_{1}$ & Height of base & $0.93 \mathrm{~m}$ \\
\hline$a_{2}$ & Length of scaffold & $1.91 \mathrm{~m}$ \\
\hline$b_{2}$ & Height of scaffold & $0.49 \mathrm{~m}$ \\
\hline$l_{1 x}$ & Length in $x$-direction from fulcrum to center of gravity (base) & $0 \mathrm{~m}$ \\
\hline$l_{1 y}$ & Length in $y$-direction from fulcrum to center of gravity (base) & $0.5 b_{1} \mathrm{~m}$ \\
\hline$l_{2 x}$ & Length in $x$-direction from fulcrum to center of gravity (scaffold) & $1.4-0.5 a_{2} \mathrm{~m}$ \\
\hline$l_{2 y}$ & Length in $y$-direction from fulcrum to center of gravity (scaffold) & $0.540 \mathrm{~m}$ \\
\hline$k_{0}$ & Virtual spring constant around fulcrum & $650 \mathrm{~N} / \mathrm{m}$ \\
\hline$m_{H}$ & Mass of virtual quality point at wire tip & $1.0 \times 10^{-6} \mathrm{~kg}$ \\
\hline$L_{t H}$ & $x$-coordinate of wire fixation point & $0.125 \mathrm{~m}$ \\
\hline$r_{H x}$ & $y$-coordinate of wire fixation point & $1.0 \mathrm{~m}$ \\
\hline$r_{H y}$ & Gravitational acceleration & $-l_{2 y}-0.5 b_{2} \mathrm{~m}$ \\
\hline$g$ & & $9.8 \mathrm{~m} / \mathrm{s}^{2}$ \\
\hline
\end{tabular}

vector, $\boldsymbol{D} \in \mathbb{R}^{5 \times 5}$ is the summary matrix of damping coefficients, and $\boldsymbol{B}(\boldsymbol{q}) \in \mathbb{R}^{5 \times 2}$ is the matrix representing the input. $\boldsymbol{A}^{T}(\boldsymbol{q}) \boldsymbol{\lambda}$ represents the restraining force that occurs between the mobile scaffold and the wire. $\boldsymbol{A}(\boldsymbol{q}) \in \mathbb{R}^{2 \times 5}$ is the matrix obtained by partial differentiation of the constraint $\boldsymbol{H}(\boldsymbol{q}, t)=0 \in \mathbb{R}^{2 \times 5}$ for the wire attachment position on the mobile scaffolding device with respect to $\dot{\boldsymbol{q}}$, and $\lambda \in \mathbb{R}^{2}$ is Lagrange's undetermined multiplier. ${ }^{(7)}$

\subsection{Kinematics model and dynamics model}

The velocity relationship equation between $\boldsymbol{q}_{S}=\left[\theta, x_{s}, x_{v}\right]^{T}$ and $\boldsymbol{q}_{H}=\left[l_{H}, \varphi_{H}\right]^{T}$ is given by

$$
\boldsymbol{q}_{H}=\boldsymbol{J}(\boldsymbol{q}) \dot{\boldsymbol{q}}_{S},
$$

where $\boldsymbol{J}(\boldsymbol{q})$ is the Jacobi matrix in the velocity relation, $\boldsymbol{\alpha}$ is the vector containing the wire tension, and $F$ is the generated force at the control target. The statics relationship is given by 


$$
\boldsymbol{F}=\boldsymbol{J}(\boldsymbol{q})^{T} \boldsymbol{\alpha} .
$$

Therefore, the kinematic model is given as follows by using the velocity vector $\boldsymbol{v}=\dot{\boldsymbol{q}}_{s}$ :

$$
\dot{q}=\left[\begin{array}{c}
I \\
J(q)
\end{array}\right] v=S(q) v
$$

In Eq. (4), $\boldsymbol{I} \in \mathbb{R}^{2 \times 2}$ is the identity matrix and $\boldsymbol{S}(\boldsymbol{q})$ is the zero-space matrix of $\boldsymbol{A}(\boldsymbol{q})$ satisfying $\boldsymbol{S}(\boldsymbol{q})^{T} \boldsymbol{A}(\boldsymbol{q})^{T}=0{ }^{\left({ }^{(8)}\right.}$ By multiplying $\boldsymbol{S}(\boldsymbol{q})$ by Eq. (1), the dynamics model is given by

$$
\bar{M} \dot{v}+\bar{C} v+\bar{K}=\bar{B} \boldsymbol{u},
$$

where $\overline{\boldsymbol{M}}=\boldsymbol{S}^{T} \boldsymbol{M S} \in \mathbb{R}^{3 \times 3}, \quad \overline{\boldsymbol{C}}=\boldsymbol{S}^{T} \boldsymbol{M} \dot{\boldsymbol{S}}+\boldsymbol{S}^{T}(\boldsymbol{V}+\boldsymbol{D}) \boldsymbol{S} \in \mathbb{R}^{3 \times 3}, \quad \overline{\boldsymbol{K}}=\boldsymbol{S}^{T}(\boldsymbol{G}+\boldsymbol{K}) \mathbf{S} \in \mathbb{R}^{3}$, and $\overline{\boldsymbol{B}}=\boldsymbol{S}^{T} \boldsymbol{B} \in \mathbb{R}^{3 \times 2}$.

\subsection{State space model}

The nonlinear state space model in this paper is given by Eqs. (4) and (5) as follows using the state variable $p=\left[v^{T}, q^{T}\right]^{T}$.

$$
\begin{gathered}
\frac{d \boldsymbol{p}}{d t}=f(\boldsymbol{p})+g(\boldsymbol{p}) \boldsymbol{u} \\
y=h(\boldsymbol{q})
\end{gathered}
$$

Equation (6) is the nonlinear state equation of the controlled object, and Eq. (7) is the output equation, and the parameters of each equation are given as follows.

$$
f(\boldsymbol{p})=\left[\begin{array}{c}
-\overline{\boldsymbol{M}}^{-1}(\overline{\boldsymbol{C}} v+\overline{\boldsymbol{K}}) \\
S(\boldsymbol{q}) v
\end{array}\right], g(\boldsymbol{p})=\left[\begin{array}{c}
\overline{\boldsymbol{M}}^{-1} \overline{\boldsymbol{B}} \\
0
\end{array}\right], h(\boldsymbol{p})=\left[\dot{\theta}, \dot{x}_{s}, \dot{x}_{v}, \theta, x_{s}, l_{H}\right]^{T}
$$

\section{Horizontal Holding Control of Mobile Scaffolding Device}

\subsection{Design of the controller}

\subsubsection{Scaffolding split controller}

The split operation of the mobile scaffolding device we are dealing with is performed at a constant speed by the operator. Therefore, we implement the manipulated variable $u_{s}$ for the scaffolding split operation using a proportional-differential (PD) speed controller, where $u_{s}$ is expressed as 


$$
u_{s}(t)=K_{P s} e_{s}(t)+K_{D s} \dot{e}_{s}(t)+G_{x}, \text { but } e_{s}=\dot{x}_{s d}-\dot{x}_{s}
$$

In Eq. (9), $K_{P s}$ is the proportional gain, $K_{D s}$ is the differential gain, $G_{x}$ is the gravity compensation term, and $\dot{x}_{s d}$ is the target value for velocity control.

\subsubsection{Horizontal holding controller}

The manipulated variable $u_{H}$ for horizontal holding control of a mobile scaffolding device is given by

$$
u_{H}(t)=K_{P H} e_{H}(t)+K_{D H} \dot{e}_{H}(t)+v_{g} \text {, but } e_{H}=l_{H d}-l_{H}
$$

In Eq. (9), $K_{P H}$ is the proportional gain, $K_{D H}$ is the differential gain, $v_{g}$ is the gravity compensation term, and $l_{H d}$ is the target value of the wire length. $v_{g}$ satisfies the following equation, where $G_{\theta}$ is the gravity term in the rotational motion of the mobile scaffolding device:

$$
\left[\begin{array}{c}
G_{\theta} \\
0 \\
0
\end{array}\right]=\boldsymbol{J}(\boldsymbol{q})^{T}\left[\begin{array}{c}
v_{g} \\
0
\end{array}\right] .
$$

\subsection{Control target}

In this paper, we design a control system that satisfies the following control target.

(a) The scaffold split motion sufficiently follows the target trajectory, which simulates the operation by the operator shown in Fig. 4. In Fig. 4, 5-25 s is a trajectory that simulates scaffolding split operations, and $35-55 \mathrm{~s}$ is a trajectory that simulates scaffolding coupling operations.

(b) The tilt of the mobile scaffolding device $\theta$ must be controlled within $\pm 1^{\circ}$ so that the workers on board the scaffold do not feel the tilt.

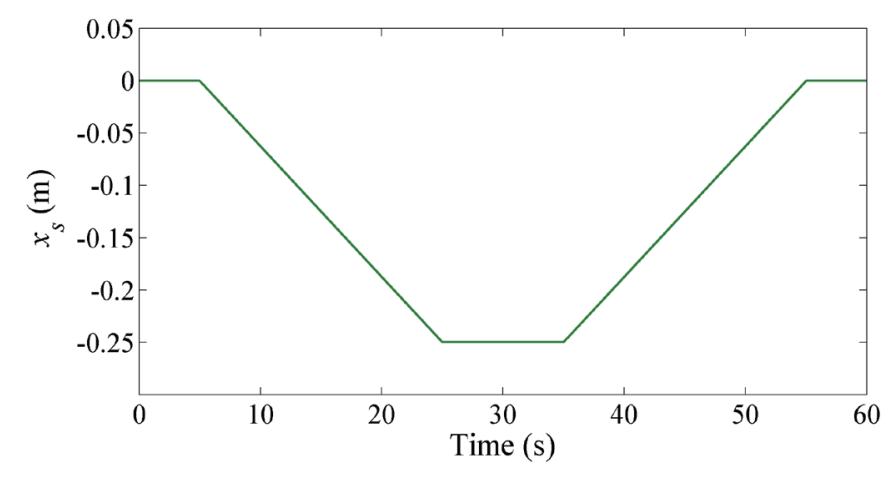

Fig. 4. (Color online) Target trajectory of mobile scaffolding device. 


\subsection{Simulation}

Figure 5 shows the simulation results for the amount of segmental movement of the scaffold, and Fig. 6 shows the simulation results for the tilt of the mobile scaffolding device. Figure 5 shows that the movement of the scaffold can follow the target trajectory shown in Fig. 4 without any steady-state deviation, and the control target for the scaffold division operation is fully satisfied. Figure 6 shows that the maximum amplitude of the mobile scaffold in the uncontrolled state is 4.3 , which was reduced to 0.03 by the wire-reeling control, satisfying the control target for horizontal retention.

\section{Damping Control with Semi-active Dynamic Absorber}

\subsection{Natural frequency of the system}

For a mobile scaffolding device that is kept sufficiently horizontal by the horizontal holding control, we obtain a new equation of motion for the rotational motion as follows. ${ }^{(9)}$

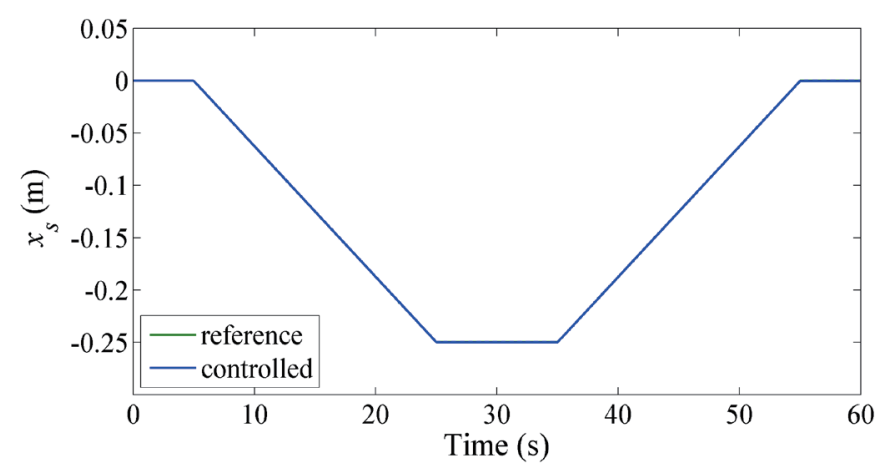

Fig. 5. (Color online) Trajectory of mobile scaffolding device.

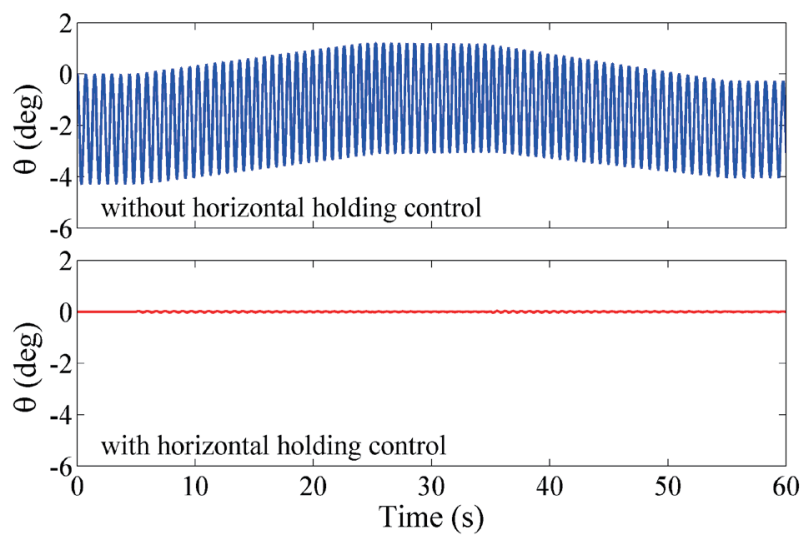

Fig. 6. (Color online) Tilt of mobile scaffolding device. 


$$
M_{\theta} \ddot{\theta}+C_{\theta} \dot{\theta}+K_{\theta} \theta+N_{\theta}=0
$$

$M_{\theta}, C_{\theta}, K_{\theta}$, and $N_{\theta}$ in Eq. (10) are expressed as follows.

$$
\begin{aligned}
& M_{\theta}=m_{1}\left(l_{1 x}^{2}+l_{1 y}^{2}\right)+m_{2}\left(l_{2 x}+x_{s}\right)^{2}+I_{1}+I_{2} \\
& C_{\theta}=\left\{2 m_{2}\left(l_{2 x}+x_{s}\right)+\frac{d I_{2}}{d x}\right\} \dot{x}_{s} \\
& K_{\theta}=m_{1} l_{1 y} g+\left\{\left(r_{H x}+x_{s}\right) \sin \varphi_{H}-r_{H y} \cos \varphi_{H}\right\} u_{H}+k_{0} \\
& N_{\theta}=m_{1} l_{1 x} g+m_{2}\left(l_{2 x}+x_{s}\right) g+u_{s} l_{2 y}+\left\{\left(r_{H x}+x_{s}\right) \cos \varphi_{H}+r_{H y} \sin \varphi_{H}\right\} u_{H}
\end{aligned}
$$

Therefore, the natural frequency $\omega_{n}$ of the mobile scaffolding device is given by

$$
\omega_{n}=\sqrt{\frac{K_{\theta}}{M_{\theta}}} .
$$

As shown in Eq. (11), the natural frequency of the mobile scaffolding device varies with the amount of movement of the scaffold $x_{S}$ and the horizontal holding input $u_{H}$. Therefore, depending on the value of $u_{H}, \omega_{n}$ may be an imaginary number. In this paper, we assume that $\omega_{n}=0$ in such a case to avoid complications in vibration control.

\subsection{Design procedure for dynamic absorber}

\subsubsection{Parameters of dynamic absorber}

The physical parameters of the dynamic absorber considered in this paper are the additional mass $m[\mathrm{~kg}]$, the spring constant $k[\mathrm{~N} / \mathrm{m}]$, the damping coefficient $c[\mathrm{~N} /(\mathrm{m} / \mathrm{s})]$ of the variable damper, and the mounting position $L[\mathrm{~m}]$ of the dynamic absorber. ${ }^{(10)}$

In this case, the natural frequency of the dynamic absorber is given by

$$
\omega_{v}=\sqrt{\frac{k}{m}}
$$

Given a mass ratio as a design parameter, the additional mass is given by

$$
m=\mu\left(m_{1}+m_{2}\right)
$$

It is also necessary to generate a large restoring force to rapidly reduce the swaying of the mobile scaffolding device. Therefore, the dynamic absorber is installed at the bottom end of the base part $\left(L=b_{1}\right)$.

The mobile scaffold to which the dynamic absorber is applied has nonlinear characteristics, as shown in Eq. (1). Therefore, the fixed-point theory design method cannot be applied, as is the 
case with conventional dynamic absorbers. In this paper, the spring constant and the damping factor are determined using the method proposed in the following discussion.

\subsubsection{Spring constant}

The frequency ratio $f$ of the mobile scaffolding device and the dynamic absorber is given by

$$
f=\frac{\omega_{v}}{\omega_{n}}
$$

From Eqs. (12) and (14), the spring constant of the dynamic absorber is given using the natural frequency $\omega_{n}$ of the mobile scaffolding device as follows.

$$
k=m\left(f \omega_{n}\right)^{2}
$$

When $x_{s}$ varies in the range of $-0.25 \leq x_{s} \leq 0$ as defined by the target trajectory in Fig. 3, the natural frequency of the mobile scaffolding device varies in the range of $0 \leq \omega_{n} \leq 9.1$. Since the natural frequencies of the mobile scaffolding device and the dynamic absorber coincide and resonate with each other when $f=1$, the range of the spring constant $k$ is determined to be $0<k$ $\leq 77.8$ in the range of $0 \leq \omega_{n} \leq 9.1$ from Eq. (15). The relationship between the frequency ratio $f$ and the amount of movement of the scaffold part $x_{s}$ is shown in Fig. 7.

As shown in Fig. 7, for $k>40$, the range of $x_{S}$ where $f>1$ is large, and the damping force by the dynamic absorber is more likely to act as an exciting force on the mobile scaffolding device. Therefore, in this paper, an arbitrary spring constant is determined in the range of $0<k \leq 40$ and given to the dynamic absorber.

\subsection{Formulation of the damping coefficient based on skyhook theory}

The fulcrum of the mobile scaffolding device and the dynamic absorber in Fig. 2 are shown in Fig. 8. In this paper, the damping coefficients of the variable damper are formulated using the skyhook theory, which is a fundamental theory of active suspensions. ${ }^{(1)}$ The conventional

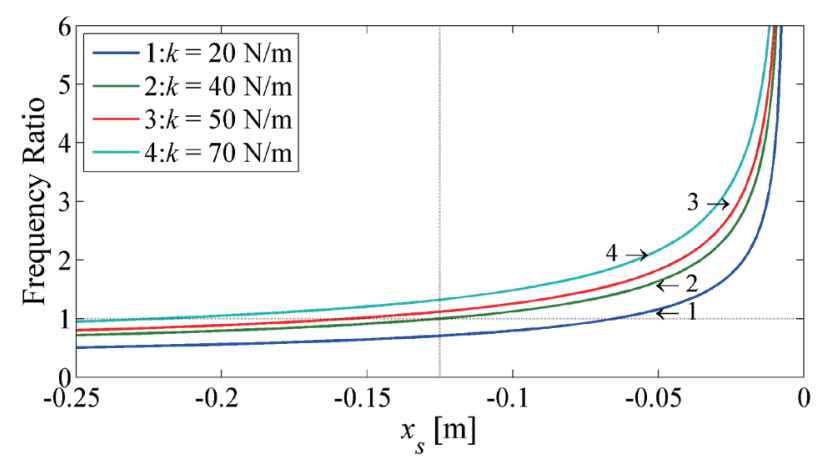

Fig. 7. (Color online) Frequency ratio. 


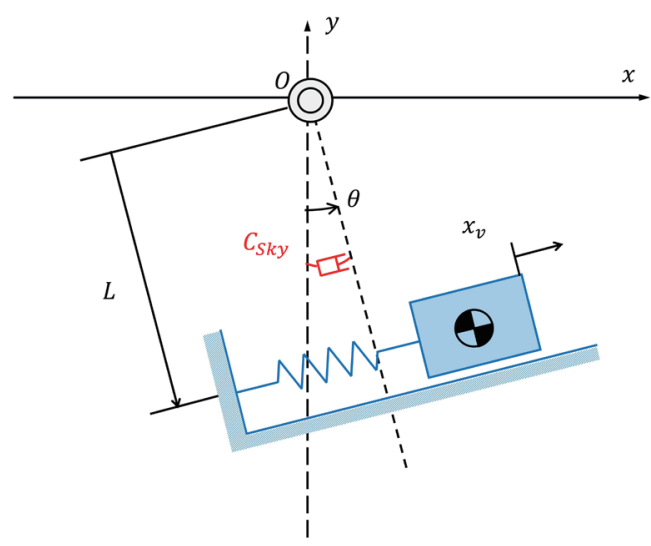

Fig. 8. (Color online) Skyhook model.

skyhook theory was proposed as a damping method for translational motion and not for rotational motion, such as that of the mobile scaffold treated in this paper. A pseudo-skyhook system for rotational motion is constructed by installing a virtual damper with a damping coefficient $C_{S K v}$ around the fulcrum of the scaffold, and the damping coefficients of the variable damper are calculated assuming that the damping force in the skyhook system and the damping force in the semi-active dynamic absorber are equivalent.

The damping force $F_{C}$ in the skyhook system shown in Fig. 8 is given by

$$
F_{C}=C_{S k y} \dot{\theta}-k x_{v} L .
$$

The damping force $F_{C}^{\prime}$ in a semi-active dynamic absorber is given by

$$
F_{C}{ }^{\prime}=-\left(k x_{v}+c \dot{x}_{v}\right) L .
$$

Considering that the damping force by the damper is the resistance to the expansion and contraction of the damper, the damping coefficient of the variable damper is given by the following equation from $F_{C}=F_{C}$ '.

$$
c=\left\{\begin{array}{cc}
-\frac{C_{S k y} \dot{\theta}}{\dot{x}_{v} L} & \left(\dot{\theta} \dot{x}_{v}<0\right) \\
0 & \left(\dot{\theta} \dot{x}_{v} \geq 0\right)
\end{array}\right.
$$

As shown in Eq. (11), the natural frequencies of this mobile scaffold cannot be uniquely determined, so $C_{S K y}$ cannot be determined analytically. Therefore, in this paper, we determine $C_{S K v}$ from the time and frequency responses of a mobile scaffolding device given an arbitrary external force. 


\subsection{Simulation conditions}

The equation of state for a mobile scaffolding device subjected to external forces is given by the following equation based on Eq. (6).

$$
\frac{d \boldsymbol{p}}{d t}=f(\boldsymbol{p})+g(\boldsymbol{p}) \boldsymbol{u}+\boldsymbol{w}
$$

The vector $\boldsymbol{w}$ for external forces in Eq. (19) is given by

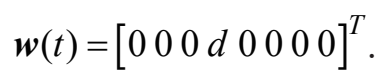

The torque due to external forces in Eq. (20) is $d[\mathrm{~N} / \mathrm{m}]$. Here, a pulse input is given to a mobile scaffold that is kept horizontal by an external force and whose amplitude is about twice the control target of the horizontal holding control, which is $\pm 1^{\circ}$, as shown in the following equation.

$$
d= \begin{cases}12.0, & 6.0 \leq t \leq 8.0 \\ 0, & \text { otherwise }\end{cases}
$$

\subsection{Simulation}

The time response of the mobile scaffolding device for the external force defined in Eq. (21) is shown in Fig. 9 and the frequency response is shown in Fig. 10. Figure 9 shows that on a scaffold without a dynamic absorber, the maximum amplitude of the vibration is $2.2^{\circ}$ at the moment the external force is applied, and the vibration continues with damping afterwards. This damping vibration is caused by the attempt to return the scaffold to the horizontal state by wire reeling, and the vibrations after $50 \mathrm{~s}$ in Fig. 9 are the steady-state error in horizontal holding control.
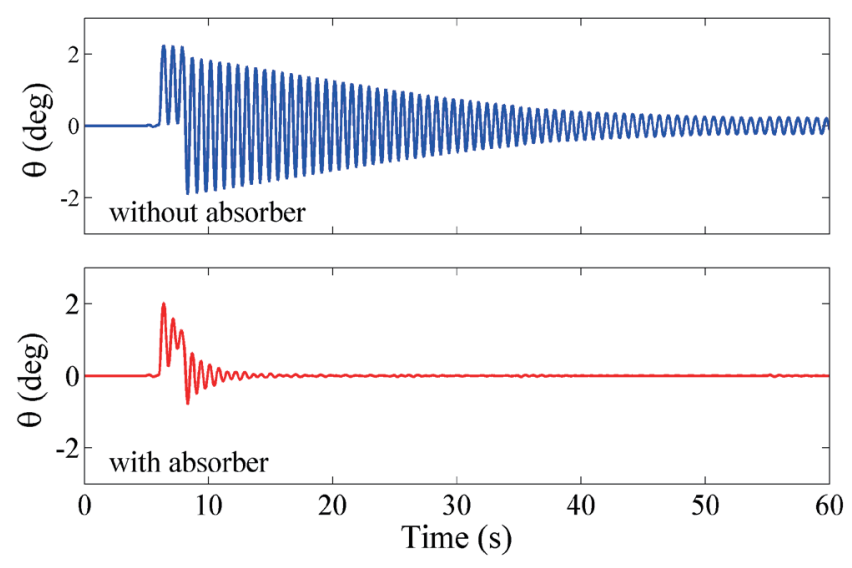

Fig. 9. (Color online) Time response of mobile scaffolding device given the pulse input. 


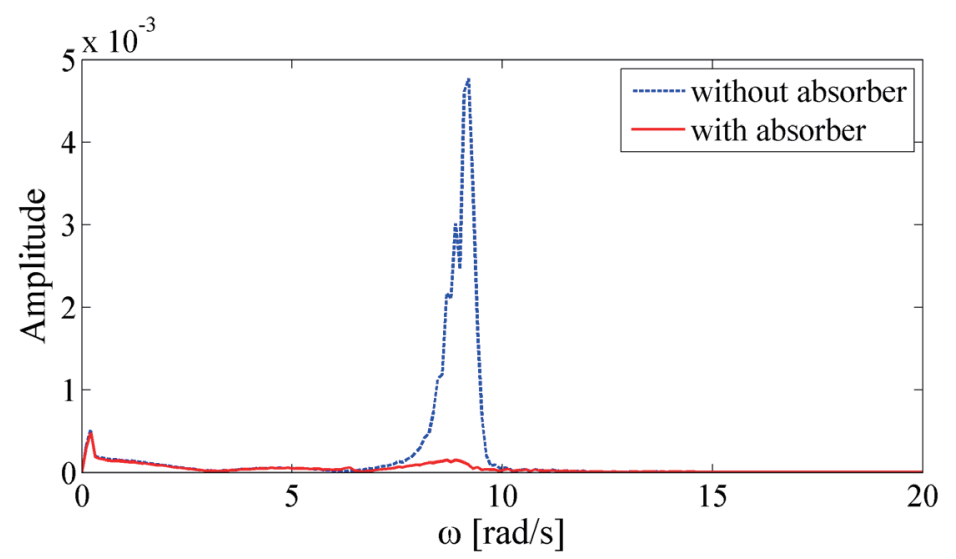

Fig. 10. (Color online) Frequency response of mobile scaffolding device given the pulse input (FFT result).

When the dynamic absorber is mounted on a mobile scaffold with the design parameters $\mu=0.10$, $k=40 \mathrm{~N} / \mathrm{m}$, and $C_{S K v}=5.0 \mathrm{Ns} / \mathrm{rad}$, the maximum amplitude of $2.0^{\circ}$ oscillation is generated at the moment the external force is applied, and then the oscillation is suppressed in about $7.0 \mathrm{~s}$ to return to the horizontal state. Figure 10 shows that the peak of the frequency generated by the external force is also well suppressed.

\subsection{Evaluation of dynamic absorber}

The parameters of the semi-active vibration absorber considered in this paper are determined so that the time and frequency responses are optimized for a vibration system given an arbitrary pulse input. Therefore, the damping performance is not guaranteed when an external force is applied, which is not defined in the design.

Here, we evaluate the damping performance of this dynamic vibration absorber by applying external forces under different conditions from those of the design.

An arbitrary random input that simulates the torque generated by an out-of-wind force is shown in Fig. 11. This random input takes the absolute value of a normal random number with a mean value of 0 and a standard deviation of 1 and is generated so that a wind force is always given in the unbounded direction of motion of the device.

Figure 12 shows the time and frequency responses of the mobile scaffold given the random torque in Fig. 11. Figure 12 shows that the maximum amplitude for the mobile scaffold without the dynamic absorber was $4.4^{\circ}$, whereas the maximum amplitude for the mobile scaffold with the dynamic absorber was $1.8^{\circ}$. This confirms that the dynamic absorber designed in this paper is effective against random external forces. Figure 13 shows that the vibration frequency peak caused by the excitation of the mobile scaffold is suppressed by the effect of the dynamic absorber. 


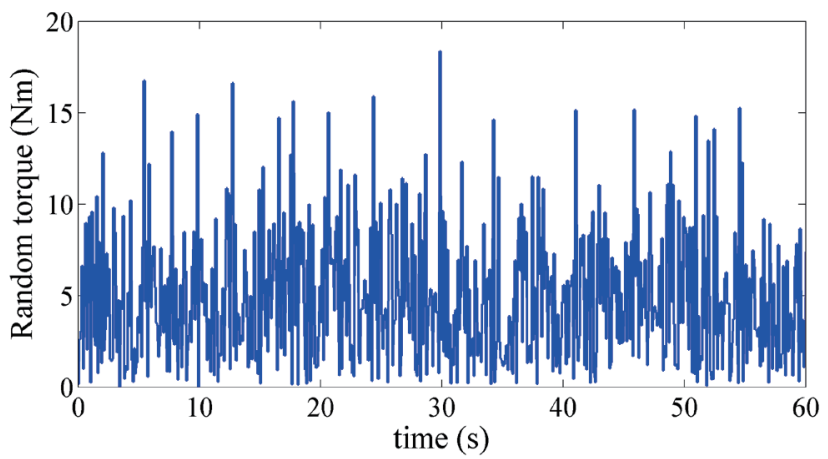

Fig. 11. (Color online) Random torque by wind force.

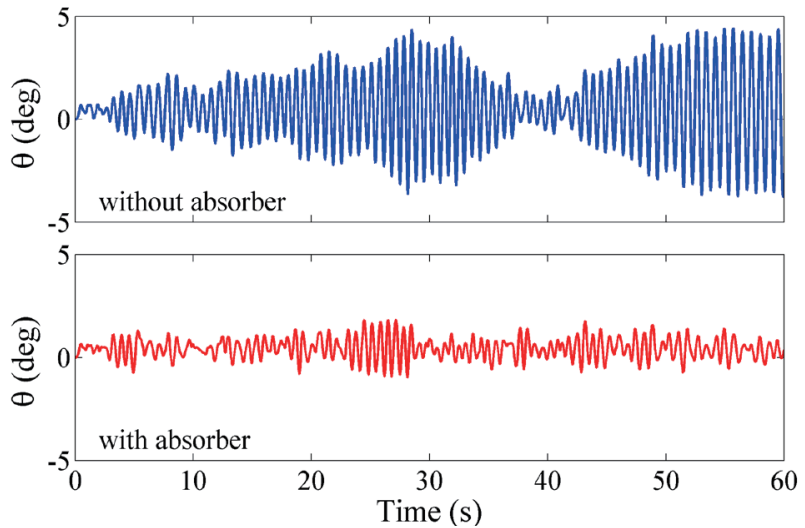

Fig. 12. (Color online) Time response of mobile scaffolding device given the random input.

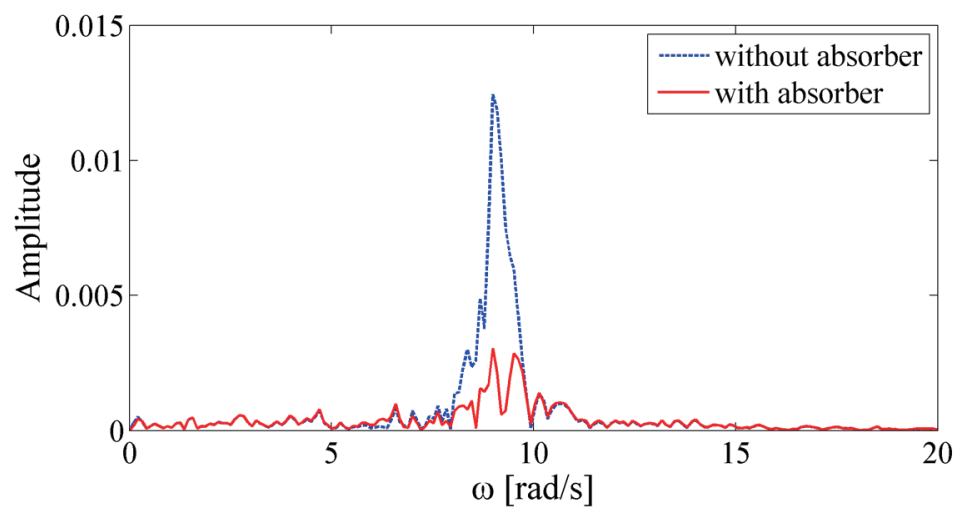

Fig. 13. (Color online) Frequency response of mobile scaffolding device given the random input (FFT result).

\section{Conclusions}

In this paper, a semi-active dynamic absorber is designed by applying the skyhook theory, which is a basic theory of active suspension, to suppress the vibration of a mobile scaffold. 
When a pulse input was applied as an external force to a mobile scaffolding device without a dynamic absorber that was sufficiently horizontal, steady-state vibration remained as a steadystate deviation under wire-reeling control, whereas the mobile scaffolding device with a semiactive dynamic absorber quickly suppressed the shaking of the mobile scaffold and returned to the horizontal state.

A mobile scaffolding device with a dynamic absorber was applied with random inputs that simulated the wind force that would be generated under the actual operating environment of the mobile scaffolding, and the maximum amplitude of the vibration was reduced to about half of what it would have been without the dynamic absorber. The semi-active dynamic absorber proposed in this paper provides damping control while monitoring the angular velocity of the rotational motion of the structure and the absolute velocity of the translational motion of the dynamic absorber in actual time. Therefore, it can be applied to a pendulum-type structure with varying vibration characteristics, such as the mobile scaffold treated in this paper. The proposed design method can be generalized for application to the damping control of pendulumtype structures and cranes.

We consider that the damping performance of the proposed semi-active absorber depends on the intensity of the pulse input given during design. Therefore, we are planning to generalize the design method as well as determine the detailed design specifications of the dynamic absorber.

\section{References}

1 Rope access technology, KISTON Corporation: http://www.rope-access.co.jp/menu-g33-lm-id142.htm (accessed February 2021) (in Japanese).

2 Bridge Inspection Vehicle Kyushu Technical Office (Kyushu Disaster Prevention and Volcanic Engineering Center), Kyushu Regional Development Bureau, Ministry of Land, Infrastructure and Transport: http://www. qsr.mlit.go.jp/kyugi/kiki_bousai/saigai_kikai/kikai_11.html (accessed November 2020) (in Japanese).

3 Rack Scaffolding Division, Yoneyama Kogyo Co.: http://www.yonekou.jp/rack (accessed November 2020) (in Japanese).

4 K. Kodama, A. Morinaga, Y. Shimomoto, I. Yamamoto, H. Matuda, and M. Matumura: Proc. 20th System Integration Division Conf. (SICE, 2019) 270 (in Japanese).

5 K. Kodama, A. Morinaga, Y. Shimomoto, I. Yamamoto, and H. Matuda: Proc. 2020 Robotics and Mechatronics Conf. (JSME, 2020) 2P1-A01 (in Japanese).

6 K. Nonami: System Dynamics and Vibration Control (Corona, Tokyo, 2010) 1st ed., pp. 62-112 (in Japanese).

7 R. M. Murray, Z. Li, and S. S. Sastry: A Mathematical Introduction to Robotic Manipulation (CRC, Florida, 1994) 1st ed., Chap. 6.

8 R. Fierro and F. L. Lewis: J. Robot. Syst. 14 (1997) 149. https://doi.org/10.1002/(SICI)10974563(199703)14:3\%3C149::AID-ROB1\%3E3.0.CO;2-R

9 M. Tagami, D. Yamaguchi, and Y. Tagawa: Trans. JSME C 78 (2012) 66 (in Japanese). https://doi.org/10.1299/ kikaic.78.66

10 H. Matsuhisa, R. Gu, Y. Wang, O. Nishihara, and S. Sato: Trans. JSME C 59 (1993) 1717 (in Japanese). https:// doi.org/10.1299/kikaic.59.1717

11 D. Karnopp, M. J. Crosby, and R. A. Harwood: J. Ind. Eng. Int. 96 (1974) 619. https://doi.org/10.1115/1.3438373 